\title{
Impact of Doxycycline on COVID-19 Patients with Risk Factors: DYNAMIC, a Multi-Centre, Randomised, Placebo-Controlled, Double-Blind Trial
}

\author{
Alexandra Poinas ( $\nabla$ alexandra.poinas@chu-nantes.fr) \\ Centre Hospitalier Universitaire de Nantes https://orcid.org/0000-0003-4183-483X \\ David Boutoille
}

Centre Hospitalier Universitaire de Nantes

Florence Vrignaud

Centre Hospitalier Universitaire de Nantes

Jean-Michel Nguyen

Centre Hospitalier Universitaire de Nantes

\section{Fabrice Bonnet}

Centre Hospitalier Universitaire de Bordeaux

Cédric Rat

Universite de Nantes

\section{Gilles Garcia}

Hopital Bicetre

\section{Anne Dompmartin}

Centre Hospitalier Universitaire de Caen

Marie-Thérèse Leccia

Centre Hospitalier Universitaire Grenoble Alpes

Lionel Piroth

Centre Hospitalier Universitaire de Dijon

\section{Eve Maubec}

Hopital Avicenne

\section{Pierre Gandon}

dermatologiste

\section{Alexandra Jobert}

Centre Hospitalier Universitaire de Nantes

\section{Soizic Boinet}

Centre Hospitalier Universitaire de Nantes

\section{Julie Cassecuel}

Centre Hospitalier Universitaire de Nantes

\section{Laurent Flet}


Centre Hospitalier Universitaire de Nantes

\section{Amir Khammari}

Centre Hospitalier Universitaire de Nantes

\section{Brigitte Dréno}

Centre Hospitalier Universitaire de Nantes

\section{Study protocol}

Keywords: COVID-19, doxycycline, patient with risk factors, outpatient, randomised controlled trial

Posted Date: January 25th, 2021

DOl: https://doi.org/10.21203/rs.3.rs-29729/v4

License: (c) (i) This work is licensed under a Creative Commons Attribution 4.0 International License. Read Full License 


\section{Abstract}

Background: The DYNAMIC study is based on three properties of tetracyclines. (1) Tetracyclines are known to chelate zinc from matrix metalloproteinases. It is possible their chelating activity may help inhibit COVID-19 infection by limiting its ability to replicate in the host. (2) As seen with dengue virus, tetracyclines may also be able to inhibit the replication of positive polarity single-stranded RNA viruses, such as COVID-19. (3) Tetracyclines are also modulators of innate immunity (anti-inflammatory activity), a property that has been used to treat inflammatory skin diseases for many years. They could therefore participate in limiting the cytokine storm induced by COVID-19. Moreover, the lipophilic nature of tetracyclines and their strong pulmonary penetration could allow them to inhibit viral replication at this level. Among the tetracyclines, doxycycline has three advantages: its long safety history (side effects are uncommon with no notable risks), its short treatment duration and its low cost.

Methods: The trial will involve 330 patients who are positive for SARS-CoV-2 infection and have one or more risk factors for worsening the disease. These patients will be included as outpatients for early treatment of illness. For logistical reasons and in order to be able to standardise the study as much as possible, recruitment will take place in 6 hospital departments covering the whole of France. For 14 days they will be given either $200 \mathrm{mg}$ of doxycycline a day or placebo. Our hypothesis is a considerable reduction in the number of patients hospitalised due to COVID-19 thanks to the treatment of doxycycline.

Discussion: This study could have an impact on the overcrowding of patients with COVID-19 at the hospital which is one of the major world-wide problems of this pandemic. This treatment would therefore contribute to supporting the deconfinement strategy by blocking the viral infection early and reducing the infectious period.

Trial Registration: On ClinicalTrials.gov, registration number NCT04371952, first published on 30 April 2020.

\section{Background}

In Wuhan, China, in the last quarter of 2019, a new coronavirus emerged, which is the third documented transmission from animals to humans[1]. Known as SARS-CoV-2 (Severe Acute Respiratory Syndrome Coronavirus 2) [2], it spread rapidly across China and many other countries [3,4]. On 11 February 2020, the World Health Organization (WHO) announced the name of the epidemic disease caused by SARSCoV-2: COVID-19 for COronaVIrus Disease 2019 (https://www.who.int/dg/speeches/detail/who-directorgeneral-s-remarks-at-the-media-briefing-on-2019-ncov-on-11-february-2020).

The latest Chinese meta-analysis performed on 43 studies involving 3,600 patients provides an overview of the clinical characteristics, laboratory results, chest imaging results, disease severity and case fatality rate of COVID-19 patients [5]. The dominant clinical features of COVID-19, which can be present in a variety of combinations, are: fever, cough, asthenia, rhinorrhoea, headache, dysgeusia, dysosmia and 
diarrhoea [6,7]. The most frequently reported laboratory abnormalities are a decreased lymphocyte count, elevated C-reactive protein (CRP) and D-dimer levels and elevated lactate dehydrogenase [6].

The vast majority of patients with COVID-19 have a good prognosis, but there are critical situations and even deaths [8]. Most of these critically ill and deceased patients did not develop severe clinical manifestations in the early stages of the disease. Some of the patients only had a mild fever, cough or muscle pain, with their condition suddenly deteriorating in the latter stages of the disease. Acute Respiratory Distress Syndrome (ARDS) and multi-organ failure occur rapidly, resulting in death within a short time [9].

The pandemic has shown that some patients may have one or more risk factors for adverse outcomes: here are the relevant characteristics identified by the French High Council for Public Health (HCSP) : 70 years of age and older, cardiovascular history, chronic respiratory disease that may undergo decompensation due to viral infection, respiratory failure, poorly controlled and/or complicated diabetes, patients with chronic renal failure on dialysis and cancer patients under treatment [10]. We also added body mass index (BMI) higher than 30 (obesity) [11-13].

The massive release of cytokines, known as a cytokine storm, is considered one of the major causes of ARDS and multi-organ failure [14]. It plays an important role in the process of worsening the disease [15]. Effective suppression of the cytokine storm is therefore an important tool in preventing the deterioration of health and saving the lives of patients with SARS-CoV-2 infection. Controlling the cytokine storm in its early stages using treatments such as immunomodulators and anti-cytokines, as well as reducing infiltration of lung inflammatory cells, is one of the keys to reducing the mortality rate of patients with COVID-19 [16]. Different therapeutics are currently being evaluated in clinical trials, although no therapy has proven its effectiveness to date.

Doxycycline is a second-generation semi-synthetic tetracycline that is chemically derived from firstgeneration tetracyclines, originally found in soil bacteria: actinomycetes [17]. Doxycycline was approved by the Food and Drug Administration (FDA) as an antibiotic in 1967 and to this day remains part of the antibiotic arsenal of most clinicians [17]. Following recent advances in knowledge of the antiinflammatory effects of doxycycline on the skin, its use has been extended in dermatology where its antiinflammatory property is now being used more than its antimicrobial property. In addition to rosacea, acne and hidradenitis suppurativa, doxycycline is used for other dermatological diseases, including bullous dermatoses, cutaneous sarcoidosis, Kaposi's sarcoma and neutrophil dermatoses (neutrophil chemotaxis) [18].

Based on these three properties of tetracyclines, we believe, like Sodhit and Etminan [19], Sargiacomo et al. [20] and Farouk and Salman [21], that doxycycline could be an effective treatment for COVID-19. The coronavirus family is known to bind to host matrix metalloproteinases (MMPs), particularly for viral survival. Tetracyclines are known to chelate zinc from MMPs [22]. It is therefore possible that their chelating activity may help inhibit SARS-CoV-2 infection by limiting its ability to replicate in the host [23]. Tetracyclines may also be able to inhibit the replication of positive polarity single-stranded RNA viruses, 
such as SARS-CoV-2. Indeed, the antiviral activity of doxycycline had already been reported against retroviruses 20 years ago, and a significant reduction in retrovirus titre has been observed after incubation of doxycycline-infected cells [24]. Other studies have shown that doxycycline inhibits the formation of dengue virus plaques by disrupting the conformational changes in the viral envelope that are necessary for virus entry [25]. They have also shown that at normal human body temperature and fever conditions, doxycycline significantly inhibited the serine protease of the virus and a concentration-dependent decrease in viral replication was observed [26].

In addition, tetracyclines are modulators of innate immunity (anti-inflammatory activity), a property that has been used to treat inflammatory skin diseases for many years. These modulatory effects are apparent on several targets of innate immunity. They can decrease the expression of NFB and the release of inflammatory cytokines such as TNF- $a$, IL-1 $\beta$ and IL-6, and inhibit the formation of inflammatory granulomas and the release of free radicals, independently of their antibiotic mechanism [18]. Furthermore, a recent publication has shown that species-independent coronaviruses induce mast cell proliferation in the respiratory submucosa, which in turn produces inflammatory agents such as histamine and protease, in addition to inflammatory cytokines such as IL-1 and IL-33 [27].

Two other studies have shown that chemically modified tetracyclines can induce mast cell apoptosis and activation of protein kinase $C$, thereby lowering the levels of inflammatory agents [28]. These teams have suggested that tetracyclines can be used to treat inflammatory disorders, including those induced by coronaviruses $[27,28]$. It should also be noted that, because of their anti-inflammatory capabilities, tetracyclines have also been documented to have in vitro activity on other viral infections such as HIV, West Nile virus and viral encephalitis [29]. Doxycycline may therefore contribute to limiting the cytokine storm induced by SARS-CoV-2. Lastly, the lipophilic nature of tetracyclines and their strong pulmonary penetration could enable them to inhibit viral replication directly at the inflammatory site.

The interest of doxycycline also lies in its long safety history (side effects are uncommon with no notable risks), its short treatment duration, which will be 14 days in our study (corresponding to the period during which SARS-CoV-2 can induce serious signs in the patient) and its low cost.

As already mentioned, it is often elderly patients with comorbidities who worsen during the second week of the disease and have to be hospitalised for respiratory deterioration or even put on mechanical ventilation. The HCSP has determined the patients with risk factors [10] who have become the target population of our study in France. We have added obesity as a risk factor since it seems to be present in the literature for COVID-19-positive patients in intensive care units [30]. Our hypothesis is to offer this treatment as soon as a patient with a risk factor is confirmed as being COVID-19-positive by a PCR test, with only a few symptoms, with no serious signs and before the onset of oxygen dependence, to reduce or even stop the cytokine storm, and thus the progression to a serious form of the disease, which will in turn avoid the need for hospitalisation.

The importance of finding medicines to keep patients at home and relieve hospital overcrowding is crucial during this pandemic. However, this outpatient trial will be carried out in hospital due to the 
required access to equipment and team facilities dedicated to clinical research.

Our study will be a multi-centre, randomised, placebo-controlled study to determine the efficacy of doxycycline in this context, by measuring the decrease in the number of patients hospitalised compared to the control arm.

\section{Methods/design}

\section{- Objectives}

The primary objective is to evaluate the decrease in the number of patients hospitalised after at least 48 hours of experimental treatment or requiring hospitalisation for COVID-19. The 48-hour delay allows a more objective attribution of the clinical course to advanced treatment. The embedded second primary objective is to evaluate the decrease in the number of patients requiring mechanical ventilator support.

The secondary objectives are to evaluate:

- The decrease in the number of patients with a SARS-CoV-2-positive PCR test 7 days after the start of the experimental treatment.

- The decrease in the total length of hospital stay.

- The decrease in the length of hospitalisation in the intensive care unit.

- The decrease in the duration of mechanical ventilator support.

- The decrease in the rate of deaths related to SARS-CoV-2 infection.

- An assessment of the safety of doxycycline.

\section{Outcomes}

The main outcome is an efficacy outcome corresponding to the percentage of patients hospitalised after at least 48 hours of experimental treatment. The outcome corresponding to the embedded second primary objective corresponds to the percentage of patients requiring mechanical ventilator support. These three outcomes are monitored in the two arms from 48 hours of treatment to 28 days corresponding to the end-of-study visit.

For the secondary outcomes:

Efficacy: These outcomes correspond to the report of the:

- Number of positive SARS-CoV-2 PCR tests at the inclusion visit and on D7.

- Total length of hospital stay. 
- Total length of hospital stay in the intensive care unit.

- Duration of mechanical ventilator support.

- Number of deaths related to SARS-CoV-2 infection.

Safety. Report of the number of adverse events (AE) and serious adverse events (SAE) over 28 days.

Apart from the first and the last secondary outcomes, the other outcomes will be recorded at 28 days. If the patient is hospitalised during the study period, this data will be recorded three months after the beginning of the treatment. For all of these secondary outcomes, percentages will be calculated.

For all of the outcomes, the data will be reported as percentages with confidence intervals and a $p$ value. Furthermore, the treatment effects, reported in the results and which meets all the outcomes except that of safety, will be estimated by risk ratio (plus confidence intervals and $p$ value). We will focus on the reduction of hospitalisations (main objective), mortality and the duration of respiratory care. Moreover, we will look at the correlation between the number of risk factors and these outcomes in the results.

\section{- Study design}

The DYNAMIC study is a superiority study of the efficacy of doxycycline. It is a prospective, phase III, randomised, stratified, double-blind, placebo-controlled, multi-centre national study to evaluate the use of doxycycline in patients with at least one risk factor for worsening COVID-19.

As the study is double-blind, the study treatment and its placebo will be managed by the pharmacist of the coordinating centre, CHU Nantes. The oral anti-inflammatory dose of $200 \mathrm{mg}$ of doxycycline or the matching placebo will be taken once a day in the evening one hour before going to bed, for 14 days. It should be noted that the placebo being used is marketed by COOPER; it is made of lactose and is very similar to the doxycycline tablet. The treatment (doxycycline or placebo) will be dispensed at the inclusion/randomisation visit. No dose adjustment is required. The treatment will end if the patient is hospitalised between day 0 (D0) and day 14 (D14).

Six French university hospitals: CHU Nantes, AP-HP [Assistance Publique - Hôpitaux de Paris; Hôpital Avicenne], CHU Bordeaux, CHU Caen, CHU Dijon and CHU Grenoble, will include 330 patients to provide at least 280 analysable patients. The inclusion period may be three to six months depending on the recruitment rate. Advertising, approved by the ethics committee, will be displayed to improve the recruitment rate. The treatment period is 14 days per patient and the last patient visit will be at 28 days (+/- 2 days) from the start of treatment.

\section{- Randomisation}


Randomisation will be stratified by centre and by severity factors ( 1 versus $\geq 2$ ). It will be performed according to a 1:1 ratio and balanced by blocks; the block size was random. The random numbers will be generated by computer. The software used to collect the data from the electronic report form allocated the patients automatically. Randomisation was performed according to the Interactive Web Response System.

Subjects are randomised into blocks as the allocation progresses, with a block being a sub-group of a predetermined size which contains a random allocation of patients. The software used for the randomisation is $\mathrm{R}$ version 9.4. An email alert will notify the investigator's team and the pharmacist that a patient has been randomised. For the pharmacist, the allocation will be revealed. The randomisation key is known only to the biostatistician and the data managers, to make it impossible for the investigator to assign a particular treatment. It should be noted that the biostatistician who carries out the randomisation is different to the biostatistician who will perform the statistical analysis.

\section{- Study population}

The study population is made up of male or female patients over 45 years of age, suspected of having COVID-19 with one or more risk factors for worsening the disease. They may be included in the study after a positive SARS-CoV-2 PCR test performed at the hospital. We chose 45 years as the minimum age in this study because this is the low end of the range for COVID-19-positive patients likely to be admitted to intensive care units [31]. In order to be eligible, as already mentioned, these patients, older than 45, must have one or more risk factors for worsening the disease as described by HCSP [10]: 70 years of age or older, cardiovascular history (stroke, coronary artery disease, complicated hypertension, cardiac surgery, NYHA class III or IV heart failure), poorly controlled insulin-dependent diabetes or complicated secondary diabetes, respiratory disease likely to undergo decompensation due to viral infection, patients with chronic renal failure on dialysis, and cancer patients under treatment. We have also added BMI $(\mathrm{BM} \mid>30)$ as a risk criterion, based on recent data from the literature [11-13,32]. Exclusion criteria are vulnerable populations and contraindication of the use of doxycycline. Box 1 presents all of the inclusion/exclusion criteria.

Furthermore, patients cannot be included in any other interventional research, unless they are hospitalised, and provided there is no drug interaction with any other experimental treatment. Patients who are hospitalised will be considered as a failed patient for the primary objective. These patients will then be followed up only for the management of this SAE. If hospitalisation occurs between D2 and D7, the PCR will not be performed at D7 and the corresponding objective will not be performed for this patient.

\section{- Study schedule}

The plan for the study described in this section is presented in Figure 1 and the flowchart in Figure 2. The screening is performed by COVID-19 emergency facilities or the COVID-19 unit of the investigating centre. 
A patient suspected of having COVID-19, with one or more characteristic clinical signs, and having one or more risk factors for worsening the disease is sent to hospital for a SARS-CoV-2 test. In the waiting room of the department specially dedicated to SARS-CoV-2 PCRs, a poster presenting DYNAMIC will be displayed. If the PCR is positive, either the patient who has seen the poster will contact the DYNAMIC team or the investigation team will contact the patient because of the PCR result. The hospital investigator explains the DYNAMIC study to the patient orally, checks the eligibility criteria, provides information on DYNAMIC and obtains the patient's signed informed consent. If the version of the informed consent changes during the patient's participation in the study, they will be informed of this immediately during the telephone calls held during the trial, and the new consent will be signed at the next consultation. The Supplementary Material file contains the French informed consent form that patients sign prior to their inclusion in the trial (the updated protocol is version 1.8 as of 28 October 2020). Furthermore, blood samples for serology and immunological markers will be collected, stored and kept for Dynamic study if the patient signed in addition the biocollection informed consent. This second informed consent form states that the samples may be used for scientific research. This biocollection and its consent procedure have been registered with French ethics committee CPP (Comité de protection des Personnes) Ouest IV under number DC-2011-1399. After a clinical examination, patients will be randomised either in the doxycycline arm or in the placebo arm. The patients, the investigators and their teams will be blind and will not know the assigned treatment. As Karanicolas et al. pointed out in their article, the biostatisticians will also be blinded until the analyses are performed to reduce the study bias [33].

On D3 and D14, a telephone call will be made by the investigator or their team. A clinical assessment of the patient will be carried out using the questionnaire shown in Figure 3.

Furthermore, the patient will answer daily this questionnaire and if their answers indicate warning signs they will call the investigators or the emergency department. If necessary, a face-to-face consultation will be conducted immediately after this call to accurately assess the extent to which the disease has worsened.

On D7, a face-to-face follow-up visit will be performed. After a clinical examination, a SARS-CoV-2 qualitative PCR test will be carried out. On D28, the face-to-face end-of-study visit will be performed. A blood sample will be kept for serology and other immunological markers. The patient will return any unused therapeutic units and the empty blister packs.

An early end-of-study visit will be carried out corresponding to the end-of-study visit (D28 visit), with the exception of serology if the patient leaves the study before 14 days. For included patients hospitalised before 28 days, the experimental treatment is stopped as soon as they are hospitalised and they are considered a failure. Clinical data related to their hospitalisation will be reported in the CRF. For patients hospitalised after 28 days and up to 90 days post-treatment, they will be followed up until discharged. This follow-up will include the collection of vigilance data and various hospitalisation data (date of end 
of hospitalisation, length of hospitalisation in an intensive care unit, duration of mechanical respiratory support, possible death).

During the trial, any drug judged necessary for the patient's well-being, which should not interfere with the evaluation of the test drug, may be administered at the investigator's discretion. Absorption of doxycycline is impaired by antacids containing aluminium and calcium, as well as magnesium, bismuth subsalicylate and iron- and zinc-containing preparations. The study drug should therefore be taken at a different time to antacids. Other prohibited treatments are those prohibited by the Summary of Product Characteristics of doxycycline and are listed in the exclusion criteria (see Box 1). However, in the absence of a change to the therapeutic management of a patient arriving at hospital according to their treatment arm, a blinding procedure is not required in this protocol. Treatment will be stopped if hospitalisation occurs.

\section{- Statistical methods}

The embedded testing strategy will be used to successively test:

- The decrease in the percentage of patients requiring hospitalisation related to SARS-CoV-2 infection after at least 48 hours of treatment.

- A decrease in the percentage of patients requiring mechanical ventilator support if the first null hypothesis is rejected.

An intermediate futility analysis will be carried out after 100 patients have been evaluated, with the objective of stopping the clinical trial if there is no probability that the null hypothesis will be rejected at the end of the full study. The sole response to this analysis is whether or not to stop the trial because of a lack of efficacy. The Peto method will be used with an alpha risk of 1 per 1,000 at the intermediate analysis and of $5 \%$ at the final analysis [34]. As already quoted, the biostatistician will be part of the blinded team. They will give the results of this intermediate analysis to the Data and Safety Monitoring Committee (DSMC), which may or may not unblind depending on the results.

The analysis will be stratified by centre and number of severity factors ( 1 versus $\geq 2$ ). The severity factors are $\geq 70$ years, $\mathrm{BMI}>30$, cardiovascular history (stroke, coronary artery disease, complicated hypertension, cardiac surgery, NYHA class III or IV heart failure), poorly controlled insulin-dependent diabetes or complicated secondary diabetes, respiratory disease likely to undergo decompensation due to viral infection, patients with chronic renal failure on dialysis, and cancer patients under treatment at the time of inclusion.

A modified intention-to-treat analysis will be applied. It will include all eligible randomised patients who provided their consent to participate in the trial. It will exclude patients who withdrew their consent prior to the assessment of the first primary endpoint, randomised patients who were incorrectly included (inclusion or exclusion criteria not met) and patients who did not receive any dose of treatment. 
A per-protocol analysis will also be performed, including all patients evaluated in their treatment group who received more than $75 \%$ of the total treatment dose.

For the main outcome (percentage of patients), we will use a Mantel-Haenszel test (stratified on centres and on the number of severity factors) to compare the rate of success (success means no hospitalisation). If the null hypothesis is rejected, we will compare the rate of mechanical ventilator between the two arms. [35,36]. For the quantitative secondary criteria, a linear model stratified on hospital centres and on the number of risk factors will be developed [37] if the minimum number of recruitments per centre allows, otherwise the model will be stratified across centres and adjusted for the number of risk factors. The statistical software used will be SAS and R, version 3.8 or later. The significance thresholds will be set at $5 \%$ in a bilateral situation.

\section{Sample size}

It is estimated that $25 \%$ of the eligible patients in the study will require hospitalisation. In the first wave, hospitalisation rates ranged from $18.1 \%$ [38] to $31.4 \%$ [31], we chose the mean. It is hypothesised that with doxycycline treatment, this rate would decrease to $12 \%$. Indeed, the article concerning the effect of doxycycline on dengue patients: "Dengue Patients Treated With Doxycycline Showed Lower Mortality Associated with a Reduction in IL-6 and TNF Levels" [39], shows that the group on doxycycline had a lower mortality rate than those in the untreated group (11.2\% [13/116] vs $20.9 \%$ [24/115], respectively, $\mathrm{p}=0.05)$. In addition, doxycycline administration resulted in a significant drop in IL-6 and TNF. These results therefore confirm our hypothesis of reducing the hospitalisation rate to $12 \%$.

An embedded testing strategy is used for the primary objective, and as the report of the European Medicine Agency stated, no adjustment is needed for multiplicity [40].

For the second embedded hypothesis, it is estimated that $23 \%$ (https://www.data.gouv.fr/fr/datasets/donnees-hospitalieres-relatives-a-lepidemie-de-covid-19/) of hospitalised patients are admitted to intensive care units. With the recruitment level we have, we will have a power of $80 \%$ to show a decrease from $23 \%$ to $10 \%$.

Under these hypotheses, with a $5 \%$ alpha risk in a bilateral situation and a power of $80 \%$, we would need to evaluate a total of 280 patients in both arms. If the first hypothesis is rejected, the power of the test of the second hypothesis would then be $83.5 \%$. Three hundred and thirty (330) patients will be included to compensate for study dropouts and non-assessable patients.

\section{Adverse event management}

For doxycycline, the main AEs expected are skin disorders (photosensitivity reaction, rash), immune system disorders (urticaria, rash, pruritus, angioedema, anaphylactic reaction) or digestive disorders (nausea, epigastralgia, diarrhoea, anorexia, glossitis, enterocolitis, and anal or genital candidiasis). There have been no reports of overdose. AEs reported for other tetracyclines following renal impairment (hepatic toxicity, hyperazotemia, hyperphosphataemia, acidosis), are unlikely to occur with doxycycline 
due to the lack of a change in blood levels in relation to the functional value of the kidney. For the placebo, the main expected effects are digestive disorders (excess gas, feeling of abdominal bloating, abdominal cramps and pain, and diarrhoea). As regards the pathology, patients with an uncomplicated viral infection of the upper respiratory tract may have non-specific symptoms such as fever, fatigue, cough (with or without discharge), anorexia, malaise, muscle aches, sore throat, dyspnoea, nasal congestion or headache. Rarely, patients may also experience diarrhoea, nausea and vomiting [6,41], and loss of taste or smell [42]. The disease can worsen to pneumonia, acute respiratory distress syndrome [43], sepsis and septic shock [44] .

All SAEs, whether expected or unexpected, require the completion of an SAE report. The investigator must ensure that the information entered in this report is accurate and clear. The SAE should be reported to the sponsor immediately (within 24 hours of being highlighted by the investigator). After receiving an unexpected SAE report, the sponsor will notify the authorities. Furthermore, a DSMC has been set up; it is a consultative committee responsible for reviewing the safety of a study on behalf of the study sponsor and coordinator/principal investigator. Members of the committee who are competent in the field of clinical trials (pathology methodology and pharmacovigilance) are not involved in the study.

The DSMC is a referral point for pharmacovigilance if an SUSAR (Suspected Unexpected Serious Adverse Reaction) or SAE poses particular analytical difficulty, or if a doubt arises concerning the risk/benefit of the study. It will also make a decision on the outcome of the futility analysis. If the study is ended early following a decision of the DSMC or the study sponsor, the regulatory authorities and the ethics committee will be informed by post within a maximum of 15 days. In any event, written confirmation will be sent to the coordinating investigator of the study (specifying the reasons for early termination) and to the principal investigator of each centre, if applicable. All patients involved in the study will be informed and will be required to attend their early discharge visit.

\section{- Ethical, regulatory and dissemination aspects}

The clinical study will be conducted in accordance with the relevant versions of the French Public Health Code, national and international good clinical practice guidelines, and the Declaration of Helsinki.

In accordance with French law, the study protocol was submitted to French regulatory authority the ANSM (Agence Nationale de Sécurité du Médicament et des Produits de Santé). This clinical study was submitted to and approved by the ethics committee of Boulogne-Billancourt (CPP Ile de France VIII) on 13 May 2020. Requests for substantial modifications should be addressed by the sponsor for approval or notification to the ANSM and/or the ethics committee concerned in compliance with Law 2004-806 of 9 August 2004 and its implementing decrees. The amended protocol will be a dated and updated version. If necessary, the information form and consent form will be amended.

All of the submissions/declarations were made by the Sponsor Department at CHU Nantes, which manages the quality of the data collected. The data collected during the study will be processed electronically in accordance with the requirements of French Data Protection Authority the CNIL (in 
compliance with French Reference Methodology MR001). Data will only be shared between the investigators. However, the datasets analysed during this study will be available from the corresponding author upon reasonable request.

An electronic Case Report Form (eCRF) shall be drawn up for each included patient. Subjects will be identified by the first letter of their family name, the first letter of their first name, the centre number and the inclusion number. This code should be the only information featured on the eCRF enabling a retrospective link to the patient. The investigator, or their team, shall also encode the patient data on any documents that may be in their possession (imaging, biology test reports, etc.) attached to the eCRF. At the end of the study, database reconciliation is carried out between the CRF database and the safety database. This reconciliation is performed before database locking. Similarly, an annual reconciliation is carried out when updating the Annual Safety Report.

As required, the sponsor has provided an insurance policy to cover the financial consequences of its civil liability in accordance with the regulations. This protocol was created thanks to a Scientific Committee. The Scientific Committee is coordinated by Professor B Dréno and its members include external and internal experts in COVID-19, experts in clinical trials and a methodologist. An inspection or audit may take place as part of this study, performed by the sponsor and/or the regulatory authorities. Inspectors will check the documents, logistics, records and any other resources the authorities consider to be associated with the clinical trial and that may be located at the trial site itself.

The trial results will be published in international, medical and scientific journals and presented at national and international conferences. The investigators will follow the rules and guidelines of the International Committee of Medical Journal Editors [45]. In practice, the Scientific Committee will be among the authors of the publication, as will the investigators who included the patients in the trial.

\section{Discussion}

The COVID-19 pandemic is affecting all continents.

Saturation of the hospital care system is feared in many countries, and the first and the second lockdown of the French population and practically the whole world was decided in an attempt to avoid hospital overcrowding.

Researchers are looking for a life-saving drug. To date, 3,904 clinical studies have been published at https://clinicaltrials.gov/ (viewed on 13 November 2020) since the beginning of the pandemic. Of these studies, 2,217 are intervention research. However, there are only 113 studies that deal with outpatient clinical research. The great strength of our study is that it tests doxycycline on at-risk patients on an outpatient basis before the cytokine wave.

In its "Clinical management of severe acute respiratory infection (SARI) when COVID-19 disease is suspected" guidance document, WHO proposes that elderly patients and those with comorbidities such 
as cardiovascular disease and type II diabetes should be hospitalised even if they do not show signs of severity. By avoiding hospitalisation, the impact of our treatment could be to relieve congestion by allowing healthcare personnel to focus on serious cases and freeing up resuscitation places. Moreover, this will prevent the need to cancel at least $20 \%$ of their usual activity in surgery and medicine, which is currently the case in French hospitals in autumn 2020.

This experimental treatment could support the deconfinement strategy by blocking the viral infection early in cases of infection, by reducing the infectious period, and by making home care possible for infected patients with a treatment whose tolerance has been proven for nearly sixty years, even in elderly patients.

The medico-economic interest is also very important. The treatment is inexpensive at around 3 euros per 15 tablets, and we hope to avoid the transfer of patients to other intensive care units, which are very expensive for the community.

Recently, in Italy, Bonzano et al. reported the benefit of doxycycline in patients with COVID-19 [46]. Their retrospective study, albeit on only six patients, suggests promising results that our randomised clinical trial will be able to strengthen by removing the bias of a retrospective study on a few cases. Furthermore, Yates et al. have presented a series of four high-risk, symptomatic, COVID-19-positive patients with known pulmonary disease, treated with doxycycline with subsequent rapid clinical improvement.

Doxycycline is an attractive candidate. If the results are as positive as we hope, this treatment could be given to the entire population, potentially for prevention of the disease.

\section{Trial status}

The updated protocol is version 1.8 as of 28 October 2020.

The first patient inclusion is expected by 15 January 2021. With an inclusion period of six months, the last patient may be included on 15 June 2021 and the study will end after their follow-up visits one month later.

\section{List Of Abbreviations}

\section{AE Adverse event}

ANSM

Agence Nationale de Sécurité du Médicament et des Produits de Santé [French National Agency for the Safety of Medicines and Health Products]

AP-HP Assistance Publique - Hôpitaux de Paris [university hospital trust of Paris

and its suburbs] 
ARS

$\mathrm{BMI}$

CNIL

protection authority]

COVID-19

CPP

CRF

CRP

D

DSMC

eCRF

FDA

HCSP

MMP

SAE

$\mathrm{SaO} \mathrm{O}_{2}$

SARS-CoV2

WHO
Agence régionale de Santé [regional health authority]

Body mass index

Commission Nationale de l'Informatique et des Libertés [French data

Coronavirus disease year 2019

Comité de protection des personnes (French ethics committee)

Case Report Form

C-reactive protein

Day

Data and Safety Monitoring Committee

Electronic Case Report Form

Food and Drug Administration

Haut Conseil de la Santé Publique [French High Council for Public Health]

Matrix metalloproteinases

Serious adverse event

Oxygen saturation

Severe Acute Respiratory Syndrome Coronavirus 2

World Health Organisation

\section{Declaration}

\section{Ethics Approval and Consent to Participate}

All patients participating in the study will be given oral and written information about this trial and will sign the informed consent form.

An independent ethics committee, the Comite de Protection des Personnes of Boulogne-Billancourt, CPP Ile de France VIII, issued a favourable opinion for this clinical trial and gave its informed consent on 28 April 2020. 


\section{Consent for publication}

The Supplementary Material file contains the French informed consent form, and information materials are available from the corresponding author on request.

\section{Availability of data and materials}

Any data required to support the protocol can be supplied on request. The data from the completed trial will not be shared and will only be transmitted to the sponsor. Data collected during the test may be processed electronically in accordance with the requirements of the CNIL (compliance with French Reference Methodology MR001).

\section{DECLARATION OF Competing interests}

The authors declare that they have no competing interests.

\section{Funding}

The "Doxycycline Versus Placebo in COVID-19-Positive Patients Without Hospitalisation Criteria: Prospective, Multi-centre, Randomised, Double-blind Study" was supported by Laboratoires Pierre Fabre, Bailleul, the Endowment Fund of CHU Nantes, Nantes Métropole and a Foundation of a French Bank (Fondation Grand Ouest de la Banque Populaire).

The pharmaceutical company Laboratoires Pierre Fabre, Bailleul played no part in the study's design, the collection, management, analysis, or interpretation of data, writing the report, or the decision to submit the report for publication.

\section{Authors' contribution}

AP; FV; AK; BD and JMN wrote the manuscript, DB; FB; CR; GG; AD; MTL; LP; EM; PG; AJ; SB; JC and LF; assisted with drafting the manuscript. BD; DB; FB; GG; AP; AK; FV and JMN designed the trial. BD; AP; AK; FV; DB; CR; SB; LF and AJ wrote the protocol and/or the file for the experimental drug and assisted with drafting the manuscript. SB coordinated the protocol's submission and follow-up with (1) the ethics committee and (2) the regulatory authorities and coordinated the trial. JMN wrote the methodological/statistical analyses in the protocol. BD; DB; FB; MTL; LP; EM and JC participated in patient enrolment and follow-up. AJ assisted with the trial's pharmacovigilance. LF wrote the experimental drug file and coordinates the provision of treatment in the centres.

All authors have read and approved the final manuscript.

Acknowledgement: In March 2020, Pierre Gandon submitted an opinion to the Nouvelle-Aquitaine French regional public health authority (Agence Régionale de Santé - ARS) regarding the potential interest of doxycycline with respect to COVID-19, having observed that acne sufferers on doxycycline escaped seasonal viral infections. 


\section{References}

1. Lu H, Stratton CW, Tang Y. Outbreak of pneumonia of unknown etiology in Wuhan, China: The mystery and the miracle. J Med Virol. 2020;92:401-2.

2. Coronaviridae Study Group of the International Committee on Taxonomy of Viruses. The species Severe acute respiratory syndrome-related coronavirus: classifying 2019-nCoV and naming it SARSCoV-2. Nat Microbiol. 2020;5:536-44.

3. Li Q, Guan X, Wu P, Wang X, Zhou L, Tong Y, et al. Early Transmission Dynamics in Wuhan, China, of Novel Coronavirus-Infected Pneumonia. N Engl J Med. 2020;382:1199-207.

4. Wang C, Horby PW, Hayden FG, Gao GF. A novel coronavirus outbreak of global health concern. The Lancet. 2020;395:470-3.

5. Fu L, Wang B, Yuan T, Chen X, Ao Y, Fitzpatrick T, et al. Clinical characteristics of coronavirus disease 2019 (COVID-19) in China: a systematic review and meta-analysis. J Infect. 2020; 80 (6): 656-665

6. Chen N, Zhou M, Dong X, Qu J, Gong F, Han Y, et al. Epidemiological and clinical characteristics of 99 cases of 2019 novel coronavirus pneumonia in Wuhan, China: a descriptive study. The Lancet. 2020;395:507-13.

7. Guan W, Ni Z, Hu Y, Liang W, Ou C, He J, et al. Clinical Characteristics of Coronavirus Disease 2019 in China. New England Journal of Medicine. 2020;382:1708-20.

8. Lai C-C, Shih T-P, Ko W-C, Tang H-J, Hsueh P-R. Severe acute respiratory syndrome coronavirus 2 (SARS-CoV-2) and coronavirus disease-2019 (COVID-19): The epidemic and the challenges. Int J Antimicrob Agents. 2020;55:105924.

9. Guo Y-R, Cao Q-D, Hong Z-S, Tan Y-Y, Chen S-D, Jin H-J, et al. The origin, transmission and clinical therapies on coronavirus disease 2019 (COVID-19) outbreak - an update on the status. Mil Med Res. 2020;7:11.

10. HCSP. Avis provisoire Recommandations relatives à la prévention et à la prise en charge du COVID-19 chez les patients à risque de formes sévères https://www.hcsp.fr/explore.cgi/avisrapportsdomaine? clefr=775 (2020) Accessed 19 Apr 2020

11. Kalligeros M, Shehadeh F, Mylona EK, Benitez G, Beckwith CG, Chan PA, et al. Association of Obesity with Disease Severity among Patients with COVID-19. Obesity (Silver Spring). 2020;28 (7):1200-4.

12. Richardson S, Hirsch JS, Narasimhan M, Crawford JM, McGinn T, Davidson KW, et al. Presenting Characteristics, Comorbidities, and Outcomes Among 5700 Patients Hospitalized With COVID-19 in the New York City Area. JAMA. 2020;323 (20):2052-9.

13. Zheng KI, Gao F, Wang X-B, Sun Q-F, Pan K-H, Wang T-Y, et al. Obesity as a risk factor for greater severity of COVID-19 in patients with metabolic associated fatty liver disease. Metabolism. 2020;108:154244.

14. Chousterman BG, Swirski FK, Weber GF. Cytokine storm and sepsis disease pathogenesis. Semin Immunopathol. 2017;39:517-28. 
15. Shimabukuro-Vornhagen A, Gödel P, Subklewe M, Stemmler HJ, Schlößer HA, Schlaak M, et al. Cytokine release syndrome. J Immunother Cancer. 2018;6:56.

16. Ye Q, Wang B, Mao J. The pathogenesis and treatment of the 'Cytokine Storm' in COVID-19. J Infect. 2020;80 (6):607-13.

17. Nelson ML, Levy SB. The history of the tetracyclines: The history of the tetracyclines. Annals of the New York Academy of Sciences. 2011;1241:17-32.

18. Henehan M, Montuno M, De Benedetto A. Doxycycline as an anti-inflammatory agent: updates in dermatology. J Eur Acad Dermatol Venereol. 2017;31:1800-8.

19. Sodhi M, Etminan M. Therapeutic Potential for Tetracyclines in the Treatment of COVID-19. Pharmacotherapy. 2020;40(5):487-8.

20. Sargiacomo C, Sotgia F, Lisanti MP. COVID-19 and chronological aging: senolytics and other antiaging drugs for the treatment or prevention of corona virus infection? Aging (Albany NY). 2020;12 (8):6511-8.

21. Farouk A, Salman S. Dapsone and doxycycline could be potential treatment modalities for COVID-19. Medical Hypotheses. 2020;140:109768.

22. Aggarwal HK, Jain D, Talapatra P, Yadav RK, Gupta T, Kathuria KL. Evaluation of role of doxycycline (a matrix metalloproteinase inhibitor) on renal functions in patients of diabetic nephropathy. Renal Failure. 2010;32:941-6.

23. Phillips JM, Gallagher T, Weiss SR. Neurovirulent Murine Coronavirus JHM.SD Uses Cellular Zinc Metalloproteases for Virus Entry and Cell-Cell Fusion. J Virol. 2017;91.

24. Sturtz FG. Antimurine retroviral effect of doxycycline. Methods Find Exp Clin Pharmacol. 1998;20:643-7.

25. Yang J-M, Chen Y-F, Tu Y-Y, Yen K-R, Yang Y-L. Combinatorial computational approaches to identify tetracycline derivatives as flavivirus inhibitors. PLoS ONE. 2007;2:e428.

26. Rothan HA, Mohamed Z, Paydar M, Rahman NA, Yusof R. Inhibitory effect of doxycycline against dengue virus replication in vitro. Arch Virol. 2014;159:711-8.

27. Kristas SK, Ronconi G, Caraffa A, Gallenga C, Ross R, Conti P. Mast cells contribute to coronavirusinduced inflammation: new anti-inflammatory strategy. J Biol Regul Homeost Agents. 2020;34 (1):914.

28. Sandler C, Nurmi K, Lindstedt KA, Sorsa T, Golub LM, Kovanen PT, et al. Chemically modified tetracyclines induce apoptosis in cultured mast cells. Int Immunopharmacol. 2005;5:1611-21.

29. Dutta K, Basu A. Use of minocycline in viral infections. Indian J Med Res. 2011;133 (5):467-70.

30. Petrakis D, Margină D, Tsarouhas K, Tekos F, Stan M, Nikitovic D, et al. Obesity -a risk factor for increased COVID-19 prevalence, severity and lethality (Review). Mol Med Rep. 2020;

31. CDC COVID-19 Response Team. Severe Outcomes Among Patients with Coronavirus Disease 2019 (COVID-19) - United States, February 12-March 16, 2020. MMWR Morb Mortal Wkly Rep. 2020;69 (12):343-6. 
32. Caussy C, Wallet F, Laville M, Disse E. Obesity is associated with severe forms of COVID-19. Obesity (Silver Spring). 2020; 28 (7):1175

33. Karanicolas PJ, Farrokhyar F, Bhandari M. Practical tips for surgical research: blinding: who, what, when, why, how? Can J Surg. 2010;53 (5) :345-8.

34. Peto R, Pike MC, Armitage P, Breslow NE, Cox DR, Howard SV, et al. Design and analysis of randomized clinical trials requiring prolonged observation of each patient. I. Introduction and design. Br J Cancer. 1976;34:585-612.

35. Mantel N, Haenszel W. Statistical aspects of the analysis of data from retrospective studies of disease. J Natl Cancer Inst. 1959;22 (4):719-48.

36. Mantel N. Chi-Square Tests with One Degree of Freedom; Extensions of the Mantel-Haenszel Procedure. Journal of the American Statistical Association. 1963;58 (303):690-700.

37. Nelder JA, Wedderburn RWM. Generalized Linear Models. Journal of the Royal Statistical Society Series A (General). 1972;135:370.

38. COVID-19 National Incident Room Surveillance Team. COVID-19, Australia: Epidemiology Report 9 (Reporting week to 23:59 AEDT 29 March 2020). Commun Dis Intell (2018). 2020;44. doi:10.33321/cdi.2020.44.29.

39. Fredeking T, Zavala-Castro J, Gonzalez-Martinez P, Moguel-Rodríguez W, Sanchez E, Foster M, et al. Dengue Patients Treated with Doxycycline Showed Lower Mortality Associated to a Reduction in IL-6 and TNF Levels. PRI. 2015;10:51-8.

40. EMA. COMMITTEE FOR PROPRIETARY MEDICINAL PRODUCTS (CPMP) - POINTS TO CONSIDER ON MULTIPLICITY ISSUES IN CLINICAL TRIALS. Available from:

https://www.ema.europa.eu/en/documents/scientific-guideline/points-consider-multiplicity-issuesclinical-trials_en.pdf (2002) Accessed 5 Nov 2020

41. Huang C, Wang Y, Li X, Ren L, Zhao J, Hu Y, et al. Clinical features of patients infected with 2019 novel coronavirus in Wuhan, China. Lancet. 2020;395:497-506.

42. Lechien JR, Chiesa-Estomba CM, De Siati DR, Horoi M, Le Bon SD, Rodriguez A, et al. Olfactory and gustatory dysfunctions as a clinical presentation of mild-to-moderate forms of the coronavirus disease (COVID-19): a multicenter European study. Eur Arch Otorhinolaryngol. 2020;

43. ARDS Definition Task Force, Ranieri VM, Rubenfeld GD, Thompson BT, Ferguson ND, Caldwell E, et al. Acute respiratory distress syndrome: the Berlin Definition. JAMA. 2012;307 (23):2526-33.

44. Rhodes A, Evans LE, Alhazzani W, Levy MM, Antonelli M, Ferrer R, et al. Surviving Sepsis Campaign: International Guidelines for Management of Sepsis and Septic Shock: 2016. Intensive Care Med. 2017;43:304-77.

45. International Committee of Medical Journal Editors (ICMJE). International Committee of Medical Journal Editors (ICMJE): Uniform Requirements for Manuscripts Submitted to Biomedical Journals: writing and editing for biomedical publication. Haematologica. 2004;89:264.

46. Bonzano C, Borroni D, Lancia A, Bonzano E. Doxycycline: From Ocular Rosacea to COVID-19 Anosmia. New Insight Into the Coronavirus Outbreak. Front Med. 2020;7:200. 
Figures

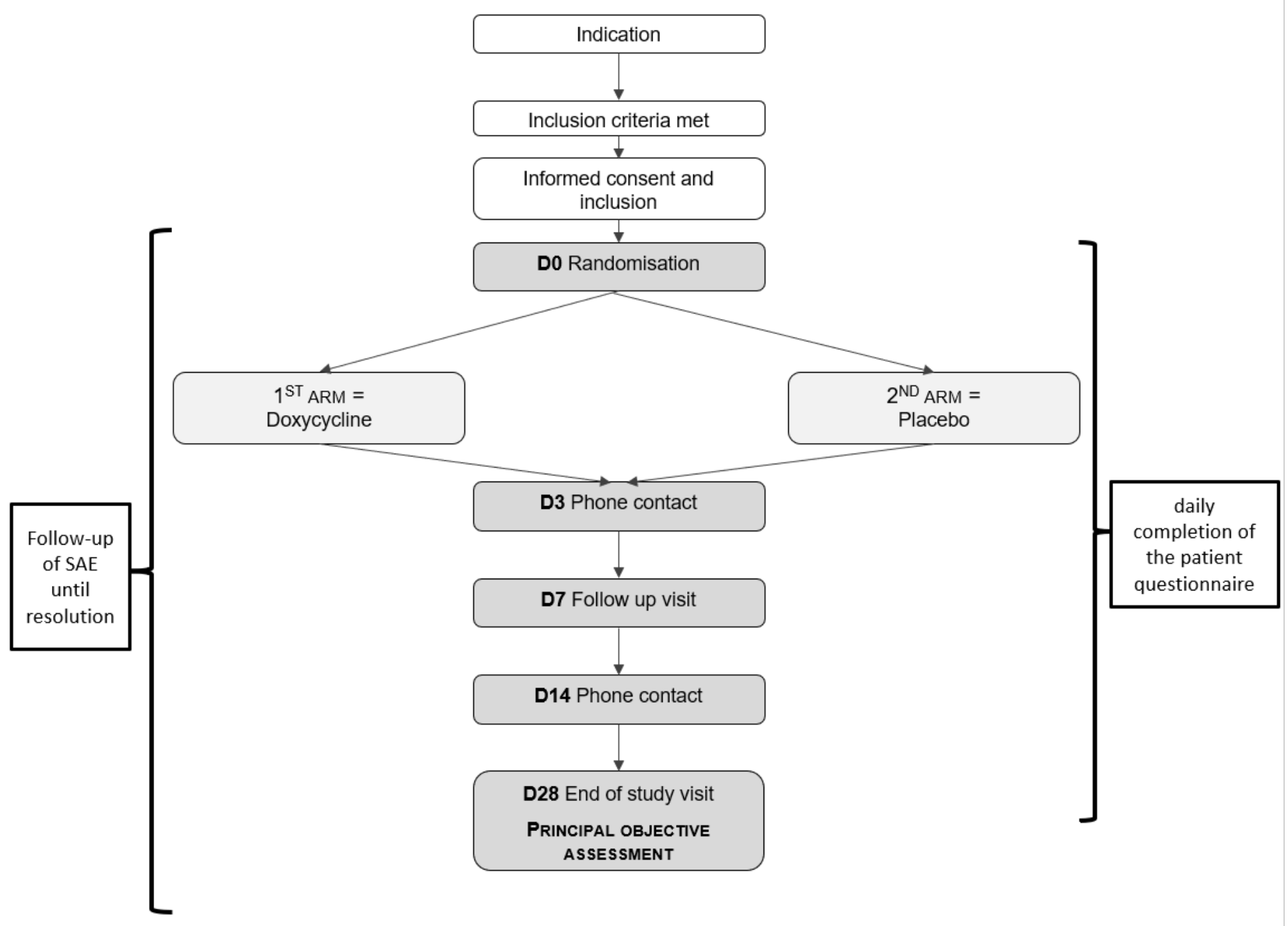

Figure 1

Study Schedule 


\begin{tabular}{|c|c|c|c|c|c|c|}
\hline STUDY PROCEDURE & SCREENING & INCLUSION VISIT & $\begin{array}{l}\text { 1ST PHONE } \\
\text { CONTACT }\end{array}$ & FoLLOW UP VISIT & $\begin{array}{l}2^{\mathrm{ND}} \text { PHONE } \\
\text { CONTACT }\end{array}$ & $\begin{array}{c}\text { END OF STUDY } \\
\text { VISIT }\end{array}$ \\
\hline VISIT DAYS & D-2 to D-1 & Do & D3 ( \pm 1 day) & D7 ( \pm 2 days) & D14 ( \pm 1 day) & D28 ( \pm 2 days) \\
\hline Patient information & $x$ & $\mathrm{x}$ & & & & \\
\hline Informed consent & & $\mathrm{x}$ & & & & \\
\hline Randomisation & & $\mathrm{x}$ & & & & \\
\hline Medical history & $\mathrm{x}$ & & & & & \\
\hline Physical examination & $x$ & $x$ & & $x$ & & $x$ \\
\hline Phone call & & & $\mathrm{X}^{\mathrm{a}}$ & & $x^{a}$ & \\
\hline SARS-CoV-2 qualitative PCR & $\mathrm{x}$ & & & $\mathrm{x}$ & & \\
\hline SARS-CoV-2 serology & & $\mathrm{x}$ & & & & $\mathrm{x}$ \\
\hline Pregnancy test & & $x^{b}$ & & & & \\
\hline Treatment dispensation & & $x^{c}$ & & & & \\
\hline Compliance request & & & & & & $\mathrm{x}$ \\
\hline Adverse events & & & $\mathrm{x}$ & $x$ & $x$ & $\begin{array}{c}\mathrm{X} \\
\text { If hospitalization, } \\
\text { follow-up until end } \\
\text { of hospitalization }\end{array}$ \\
\hline
\end{tabular}

a Questionnaire filled in by the patient daily basis in the patient-notebook with the monitoring of treatment compliance

b PCR carried out in routine care before DO

c After a negative pregnancy test result

\section{Figure 2}

Flowchart Study 


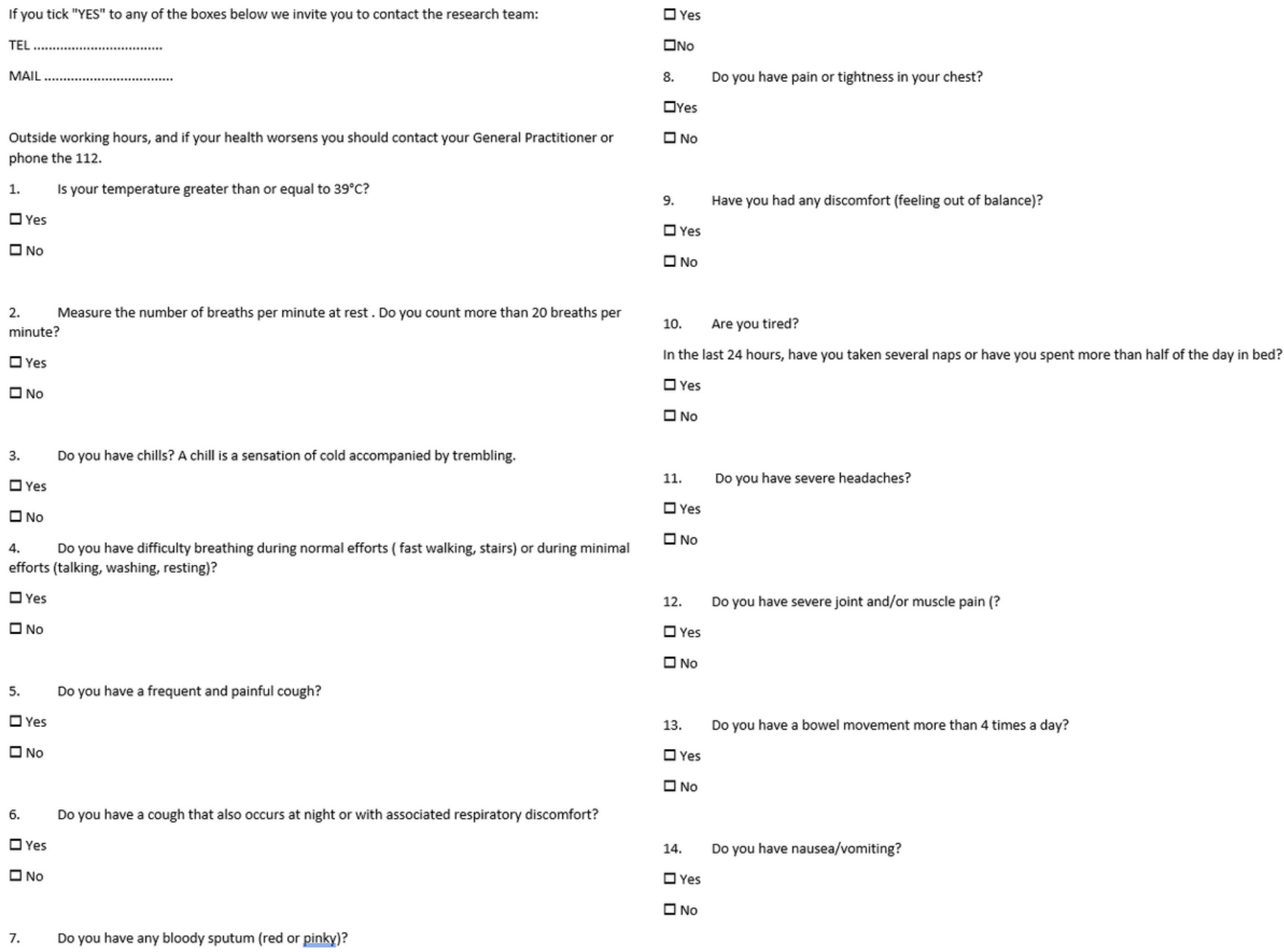

\section{Figure 3}

Patient clinical re-evaluation questionnaire (D3 and D14) and daily patient self-reporting questionnaire

\section{Supplementary Files}

This is a list of supplementary files associated with this preprint. Click to download.

- Box1revised.docx

- SPIRITDYNAMICrevised.doc

- s1.docx

- EthicCommiteeapprobationtranslatedversion.pdf

- EthicCommiteeapprobationFrenchversion.pdf

- Informedconsent.pdf

- Fundingdocumentation.doc 